\title{
Editorial
}

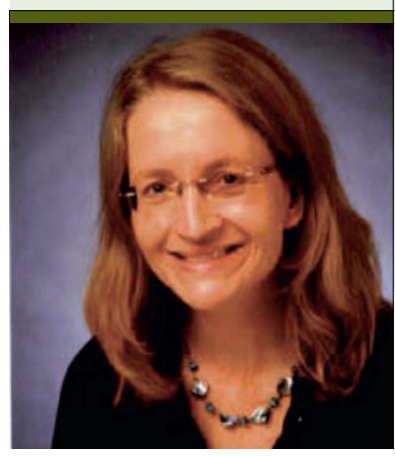

"Gerade ältere Schmerzpatienten profitieren von

körperlich-aktivierender Therapie"

Dr. Dipl.-Psych. Corinna Leonhardt

Institut für Medizinische Psychologie am Fachbereich Medizin der

Philipps-Universität Marburg

\section{Mit Bewegung gegen Schmerz}

$\mathrm{K}$ örperlich-aktivierende Übungen wirken sich positiv auf Schmerzverringerung, körperliche Fähigkeiten und Stimmungsverbesserung aus. Allen chronischen Schmerzpatienten kann mit guter wissenschaftlicher Evidenz ein körperliches Aktivitätsprogramm empfohlen werden. Die Amerikanische Geriatrische Gesellschaft (AGS) und auch die Australische Schmerzgesellschaft plädieren sogar in ihren Leitlinien für eine körperlich aktivierende Therapie bei älteren Schmerzpatienten.

Durch regelmäßige körperliche Aktivität wird nicht nur die Schmerzbewältigung verbessert, auch das Herz-Kreislauf-System, der Immunstatus und die kognitiven Fähigkeiten werden positiv beeinflusst. Der ältere Mensch bleibt im Alltag länger leistungsfähig und selbstständig.

Aber gerade älteren Menschen mit Schmerzerkrankungen fällt es oft schwer, sich für Bewegungsprogramme und eigenständige Übungen zu begeistern. Die Angst vor erneuten Schmerzattacken durch bestimmte Bewegungen oder die Angst zu stürzen und damit weiteres Leid zu erfahren, beherrscht oft den Alltag. Dass dabei aber nicht nur die Ängste der Betroffenen selbst eine Rolle spielen, sondern auch die Einstellungen und Ängste der sie betreuenden Personen - Angehörige, Pflegekräfte und auch Ärzte, haben kanadische Kollegen herausgefunden. Untersuchung von Theresa G. Fitzgerald und ihren Kollegen ergaben, dass sich die Ängstlichkeit der Pflegepersonen bezüglich Schmerz oder Sturz direkt auf das Verhalten der betreuten Menschen auswirkt [u.a. Fitzgerald TG et al. Gerontology. 2009; 55(4):460-7].

Wie kann körperlich-aktivierende Therapie bei älteren Schmerzpatienten gelingen? Ein wesentlicher Aspekt: Positive Stimmungen bei körperlichen Aktivitäten sind stärker motivierend als gesundheitsrelevante Argumente! Ärzte und Pflegepersonal, wie auch Physiotherapeuten können wichtige Motivatoren sein und gemeinsam mit den Angehörigen die Patienten anleiten. Allerdings nicht mit Ver- und Geboten. Wichtig ist es nach meiner Erfahrung, individuell abgestimmte und alltagsrelevante Bewegungsabläufe zu etablieren. Dabei hilft ein Gesprächsstil, der sich an die motivierende Beratung (Motivational Interviewing), wie sie William R. Miller und Stephen Rollnick in ihrem Buch „Motivierende Gesprächsführung " [Lambertus Verlag, 2009] beschreiben, anlehnt. Es gilt, die „intrinsische Motivation“ des Patienten zu stärken. Bei der konkreten Ausgestaltung von Bewegungsprogrammen für den älteren Schmerzpatienten ist gerade seine besondere Situation aufgrund des Alters zu beachten (z.B. Schriftgröße bei Materialien, beginnende kognitive oder Sinnes-Einschränkungen in der Kommunikation, Transportprobleme, Bedürfnis nach sozialem Miteinander).

Besonders im ambulanten und Primärversorgungsbereich scheint es einen starken Nachholbedarf für konkrete Motivierungs- und Therapiekonzepte älterer Schmerzpatienten zu geben. Eine gute interdisziplinäre Abstimmung zwischen Ärzten, ggf. Pflegepersonen und Physiotherapeuten ist meiner Einschätzung nach für eine bewegungsorientierte Schmerztherapie wichtig. Wir alle sollten dem Schmerzpatienten und insbesondere dem älteren vermitteln:

— Sie können mit Bewegung etwas gegen Ihre Schmerzen tun!

_Die Erhaltung Ihrer körperlichen und geistigen Beweglichkeit und Ihre Lebensqualität ist unser gemeinsames Ziel!

_Es lohnt sich!

Ihre Corinna Leonhardt

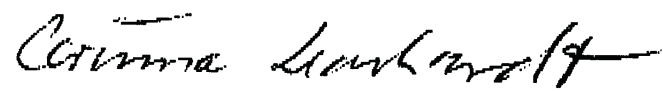

